modèles F....:!

ling uistiques

\section{Modèles linguistiques}

$59 \mid 2009$

Génétique de la production écrite et linguistique

\title{
Manuscrits de travail et linguistique de la production écrite
}

Jean-Louis Lebrave

\section{OpenEdition}

Journals

Édition électronique

URL : https://journals.openedition.org/ml/330

DOI : $10.4000 / \mathrm{ml} .330$

ISSN : 2274-0511

Éditeur

Association Modèles linguistiques

Édition imprimée

Date de publication : 1 janvier 2009

Pagination : 13-21

Référence électronique

Jean-Louis Lebrave, "Manuscrits de travail et linguistique de la production écrite », Modèles

linguistiques [En ligne], 59 | 2009, mis en ligne le 04 avril 2013, consulté le 01 juillet 2021. URL : http:// journals.openedition.org/ml/330 ; DOI : https://doi.org/10.4000/ml.330 


\section{Manuscrits de travail et linguistique de la production écrite}

Jean-Louis Lebrave

\section{La substance sémiotique du manuscrit de travail excède les possibilités du traitement linguistique.}

L'apport de la linguistique dans l'appréhension scientifique des brouillons ${ }^{1}$ a été double. D'abord, elle a fourni des outils qui ont permis $\mathrm{d}^{\prime}$ « apprivoiser » les brouillons. Le premier de ces outils est d'ailleurs la notion de variante héritée de la philologie dans sa dimension éditoriale. La linguistique structurale a de même apporté la notion de paradigme. Et il a suffi d'adapter les opérations de commutation en leur ajoutant l'orientation temporelle pour disposer de la substitution orientée dans un contexte. Dans un second temps, les théories de l'énonciation ont permis d'appréhender avec rigueur ce qui, dans le foisonnement des brouillons, relève de l'inscription du sujet parlant/écrivant dans ses discours. Mais la linguistique a aidé aussi les chercheurs à trouver l'audace d'explorer le continent de la genèse : audace de s'attaquer à des données qui défiaient a priori les possibilités d'une linguistique cantonnée au niveau de la phrase et peinant à appréhender ce que J. Fourquet appelait la « linguistique au sens large ", en particulier la psycholinguistique ; audace de mêler sa voix au concert des littéraires et de prétendre qu'on avait des choses aussi intéressantes à dire sur l'écriture de Proust ou de Flaubert que les spécialistes de Proust et de Flaubert.

Mais en même temps, il est apparu rapidement ${ }^{2}$ que les brouillons excèdent le traitement linguistique, tout simplement parce qu'ils comportent en abondance des données non linguistiques. On peut appréhender celles-ci de plusieurs manières. On peut y voir les traces du processus de production, inscrites comme des scarifications sur le corps

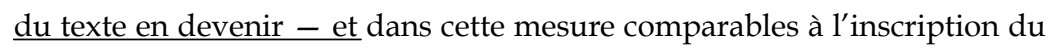

1. Sur la question des rapports entre la critique génétique et la linguistique, voir aussi A. Grésillon et J.-L. Lebrave, « Linguistique et génétique des textes : un décalogue ». Le français moderne, numéro spécial : «Tendances actuelles de la linguistique française » Paris, CILF, 2008, p. 37-49.

2. Cf. par exemple J.-L. Lebrave, « Lecture et analyse des brouillons ». Langages 69, mars 1983, p. 11-23. 
sujet parlant dans le système de la langue à travers le système de la personne, les temps verbaux, etc. On peut aussi les appréhender comme la mémoire d'une temporalité qui est celle, non du texte lui-même, mais de sa genèse. On les décrira alors comme la projection du temps de l'écriture sur la ligne du temps de l'écrit, et on s'efforcera de convertir ces données topographiques en informations chronologiques sur le déroulement de la genèse textuelle. On peut encore y reconnaître la mise en œuvre d'un système partiellement conventionnel de marquages de l'écrit : biffures, inscriptions dans les marges du déjà écrit, becquets divers, traits, etc. Enfin, et c'est à n'en pas douter leur trait le plus saillant, on peut les considérer comme les caractéristiques individuelles qui constituent la signature personnelle du scripteur, tout ce qui contribue à faire qu'on reconnaît au premier coup d'œil, sans avoir besoin de le lire, un manuscrit de Proust ou de Flaubert qu'on ne connaît pas encore. Toujours fragmentaire, cette familiarité avec l'inscription quasi charnelle du scripteur dans son brouillon indépendamment de toute référence à son contenu textuel est précieuse, notamment lorsqu'on déchiffre le manuscrit. C'est elle qui permet de redonner vie à tous les signes déposés dans la page manuscrite.

Dans sa singularité absolue et son foisonnement toujours renouvelé, cette matière sémiotique a toujours fasciné les généticiens. Ils se sont efforcés de la domestiquer en l'analysant ${ }^{3}$. Mais elle échappe fondamentalement à toute approche exhaustive. C'est que cette substance de l'expression (pour reprendre l'expression que j'avais utilisée dans un petit texte en hommage à J. Fourquet ${ }^{4}$ ) contient des éléments irréductibles à toute linguistique. On peut, mutatis mutandis, faire un parallèle avec la substance sonore de la parole, qui se laisse pour une part réduire par la phonologie - et même par une phonétique descriptive - mais dont une part est nécessairement laissée de côté par la linguistique.

\section{La construction des opérations fondamentales}

Néanmoins, le croisement des données verbales et de ce qu'on peut formaliser et structurer de la substance de l'expression a permis de construire des opérations dont les brouillons sont le théâtre.

D'abord, au niveau le plus général de la page, on identifie contrastivement des zones qui portent des données graphiques et des zones restées vierges. Généralement, ce découpage permet d'identifier des

3. Cf. notamment L. Hay (éd), De la lettre au livre. Sémiotique des manuscrits littéraires. Paris, Éditions du CNRS, 1989. Cf. aussi le numéro 10, « Sémiotique », de la revue Genesis (1994), édité par L. Hay.

4. «Les manuscrits entre la substance de l'expression et la substance du contenu ». Colette Cortès et A. Rousseau (éd), Catégories et connexions. En hommage à Jean Fourquet pour son centième anniversaire. Villeneuve d'Ascq, Presses universitaires du Septentrion, 1999, p. 371-378. 
lignes, des interlignes, des marges, un pied de page, etc. On isole ensuite des assemblages de données graphiques qu'on interprète comme des traces d'opérations. Une forme graphique déchiffrable à laquelle vient se surajouter un trait qui la traverse sera réinterprétée comme une suppression. Si à ce premier groupe de données graphiques vient s'associer une autre forme graphique déchiffrable qui, du point de vue linguistique, peut être interprétée comme remplaçant la première, on interprètera l'ensemble comme un remplacement. Si le manuscrit comporte une forme graphique déchiffrable placée à l'extérieur des lignes, soit dans l'interligne, soit dans les marges, soit encore sur un autre feuillet, mais à laquelle d'autres signes graphiques sont associés qui indiquent qu'elle doit s'insérer à l'intérieur d'une forme graphique déchiffrable préexistante, on interprètera l'ensemble comme un ajout. Insistons sur le fait que seule l'information linguistique donne un statut aux données visuelles, mais qu'inversement les données visuelles sont ininterprétables sans les données linguistiques.

C'est de cette manière que les généticiens ont été amenés à poser l'existence de quatre opérations fondamentales : l'ajout, la suppression, le remplacement, le déplacement. Et à regrouper l'ensemble sous le nom de substitution orientée dans le temps :

$\mathrm{A} \rightarrow \mathrm{B}$ n'est pas identique à $\mathrm{B} \rightarrow, \mathrm{A} \rightarrow 0$ n'est pas identique à $0 \rightarrow \mathrm{A}$, etc.

Il importe de préciser que ces opérations sont encadrées par un contexte stable à l'intérieur duquel elles prennent sens. La notation $\mathrm{A} \rightarrow \mathrm{B}$ est la forme abrégée de : XXX / A $\rightarrow$ B / YYY, etc.

Il convient encore de rappeler que l'unification des quatre opérations élémentaires sous l'opération unique de substitution a été suggérée par les contraintes liées au traitement informatique des données (il fallait réduire au maximum le coût du traitement en temps de calcul) et aussi par l'influence des préférences « esthétiques » des mathématiques pour la simplicité des généralisations pertinentes.

Cette méthode de structuration des données manuscrites - c'est le titre d'une « action thématique programmée » (ATP) du CNRS dont j'avais obtenu le financement en 1979 - est efficace. Elle m'a permis par exemple de transcrire sous forme $\mathrm{d}^{\prime}$ « édition chronologique » l'ensemble du dossier manuscrit de la Lutezia de $\mathrm{H}$. Heine et de construire un dictionnaire de toutes les substitutions que ces brouillons contiennent. Plus généralement, la notion de substitution orientée par une chronologie reste un bon outil pour appréhender un certain nombre de phénomènes observables dans les brouillons.

Mais ses limites sont apparues rapidement. La première de ces limites a un rapport direct avec la problématique du traitement linguistique. J'ai souligné que la substitution n'a de sens qu'à l'intérieur d'un contexte stable : pour exister et pour être perçue, la variation doit se détacher sur un fond invariant. Or, s'il est toujours possible d'identifier le contexte gauche d'une opération, il n'en va pas de même du contexte droit. Ce n'est 
possible que pour ce que nous avons appelé dans nos premiers travaux les corrections «tardives » ou « de relecture » - il serait plus juste de dire, en employant une définition négative, les corrections "qui ne sont pas immédiates ", c'est-à-dire celles qui apparaissent graphiquement comme venant modifier des données textuelles déjà existantes. En revanche, les corrections «immédiates » ou " au fil de la plume » sont privées de contexte droit, puisque celui-ci ne pourrait être constitué que par la totalité des suites virtuelles possibles à ce qui était déjà écrit au moment où le scripteur effectue une correction. C'est d'ailleurs ce qui distingue les manuscrits de travail des autres documents comportant des textes variants.

À cette difficulté, j'ai apporté une solution «opportuniste » et une solution plus " génétique ». La solution opportuniste consiste à interrompre arbitrairement le fil temporel de la production écrite à chaque ponctuation forte. De cette manière, on évite une cascade sans fin d'opérations dont chacune se serait emboîtée dans la précédente, sans qu'on puisse jamais refermer l'ensemble autrement que par le point final éventuel. La solution génétique prend appui sur le fait que chez tous les scripteurs, la production est rythmée par des phases d'interruption, de sorte qu'on peut identifier des périodes de travail. C'est du moins le cas pour les deux corpus que j'étudiais lorsque j'ai mis la notion de substitution en pratique, celui de Lutezia et celui des premiers brouillons de la Recherche du temps perdu. D'où ce que j'ai appelé des "blocs génétiques » dans la transcription du Cahier 3 de Proust reproduit dans Proust à la lettre.

Ces deux solutions relèvent certes $\mathrm{du}$ « bricolage », mais elles restent acceptables. Il n'est pas aberrant de supposer que le texte en cours de rédaction n'existe pas sous forme achevée (c'est-à-dire entièrement fixée dans sa forme syntaxique et dans ses choix lexicaux) au-delà de la phrase en train d'être écrite, et qu'au-delà de cette frontière, on entre dans le domaine d'une « intention de communication » plus vague, et encore incomplètement actualisée. De même, il est certain que toute écriture (du moins d'un texte un peu long) est découpée en périodes de travail successives séparées par des ruptures temporelles. En revanche, la seconde limite à l'appréhension des brouillons en termes de substitutions apparaît immédiatement comme rédhibitoire. Dès qu'on s'attaque à des manuscrits complexes, comme ceux de Flaubert, il devient rapidement évident qu'une reconstitution exhaustive de toutes les opérations, dans l'ordre où elles sont apparues, est impossible. Les données graphiques sont si complexes et imbriquées les unes dans les autres qu'elles autorisent le plus souvent une pluralité d'hypothèses sur « ce qui s'est réellement passé » pendant le processus d'écriture. Il devient dès lors illusoire de vouloir donner une liste exhaustive de toutes les opérations d'écriture et de réécriture. En revanche, la reconstitution partielle d'opérations « locales » dans un fragment de manuscrit reste parfaitement possible, et 
même indispensable, notamment lorsqu' on veut suivre, du début de la genèse à la fin de l'écriture, l'histoire d'un fragment donné, ou l'évolution $\mathrm{d}^{\prime}$ une expression particulière ${ }^{5}$.

C'est pour échapper à cette aporie que les linguistes de l'ITEM ont fini par recourir presque exclusivement à la «transcription diplomatique ». On le sait, celle-ci transcrit toutes les données verbales de manière intégrale, mais ne retient de la substance graphique que l'ensemble des données topographiques, et le cas échéant certaines données liées au ductus et au rythme de l'écriture. On voit bien que par cette démarche, on reste en quelque sorte en deçà du travail de reconstruction des opérations : si un fragment textuel se trouve dans l'interligne au-dessus d'un fragment textuel biffé, on se contente de reproduire cette disposition graphique, sans l'interpréter comme un remplacement.

\section{Génétique, philologie, variantes}

Je voudrais clore cette mise en perspective de la notion de substitution génétique par une remarque. Qu'il s'agisse de la difficulté liée à la définition du contexte droit des substitutions dans le cas de corrections " au fil de la plume », ou de l'impossibilité de reconstituer de manière fiable l'histoire des opérations d'écriture au sein d'un manuscrit complexe, la difficulté me paraît tenir dans les deux cas à ceci : on voudrait appréhender le processus d'écriture tel qu'il s'est déroulé réellement, mais celui-ci ne nous est accessible qu'à travers des produits : les énoncés (ou fragments d'énoncés) qui sont le résultat de ce processus, et les traces graphiques que le processus lui-même a laissées derrière lui.

Le traitement par les substitutions ne peut donc faire autre chose que comparer entre eux des états successifs du texte en devenir, et chercher quelles lois président aux transformations que cette comparaison permet d'identifier. Il ne peut s'agir que d'une génétique des transitions entre états, non d'une appréhension du processus de production en tant que telle. On est en réalité confronté au même paradoxe que celui que dénonçait

5. Pour un exemple de cette démarche, cf. la manière dont nous avons suivi le parcours de l'expression « quatre vallées larges et profondes » depuis les notes prises par Flaubert dans ses ouvrages sur la Palestine jusqu'au texte final d'Herodias. Cf. A. Grésillon \& J.-L. Lebrave, « Flaubert : Ruminer Herodias ». D. Ferrer et J.-L. Lebrave (éd), L'écriture et ses doubles. Genèse et variation textuelle. Paris, 1991, Ed. du CNRS, pp. 27-109. Cf. aussi les analyses qu'I. Fenoglio a menées sur la genèse des autobiographies d'Althusser. I. Fenoglio, «Énonciation et genèse dans les autobiographies d'Althusser. Deux récits séparés - de sa rencontre avec Hélène ». Genesis n 17, 2001, p. 131-150. I. Fenoglio, «Une photo, deux textes, trois manuscrits. L'archivage linguistique d'un geste d'écriture identifiant ». Langages, $n^{\circ} 147,2002$, Processus d'écriture et marques linguistiques. Nouvelles recherches en génétique du texte (I. Fenoglio et $\mathrm{S}$. Boucheron-Pétillon éd.), p. 56-69. 
T. Todorov en 1970 à propos de l'énonciation : nous n'avons jamais accès qu'à des énonciations toujours déjà énoncées. De même, nous ne connaissons du processus que les produits qu'il laisse derrière lui.

Dès lors, à partir du moment où on appréhende les données de la génétique des textes sous forme de données de langue, il est vain de prétendre, comme certains généticiens sont parfois tentés de le faire, à une originalité absolue du matériau génétique. Il n'y a pas de différence radicale entre des états textuels variants et la variation qu'on peut observer dans les brouillons. (En revanche, il y a généralement une différence de degré, le foisonnement de la variation étant souvent beaucoup plus intense dans les brouillons.) On peut donc réconcilier la philologie et la génétique et unifier le panorama de la variation textuelle, comme j'ai tenté de le faire à l'aide du tableau suivant dans la synthèse que j'ai rédigée avec J.-G. Ganascia à l'occasion de l'école thématique de critique génétique organisée par l'ITEM en 20046. Dans ce tableau, la phase 1 correspond à une gestation avant toute trace textuelle, la phase 2 à la première mise en texte, souvent éclatée et fragmentaire, la phase 3 aux ajustements, transformations, réécritures effectués par l'auteur avant la première mise en circulation du texte, la phase 4 aux transformations effectuées par l'auteur après la mise en circulation du texte, et la phase 5 aux altérations postérieures à la dernière édition autorisée, et donc à toutes les transformations non auctoriales.

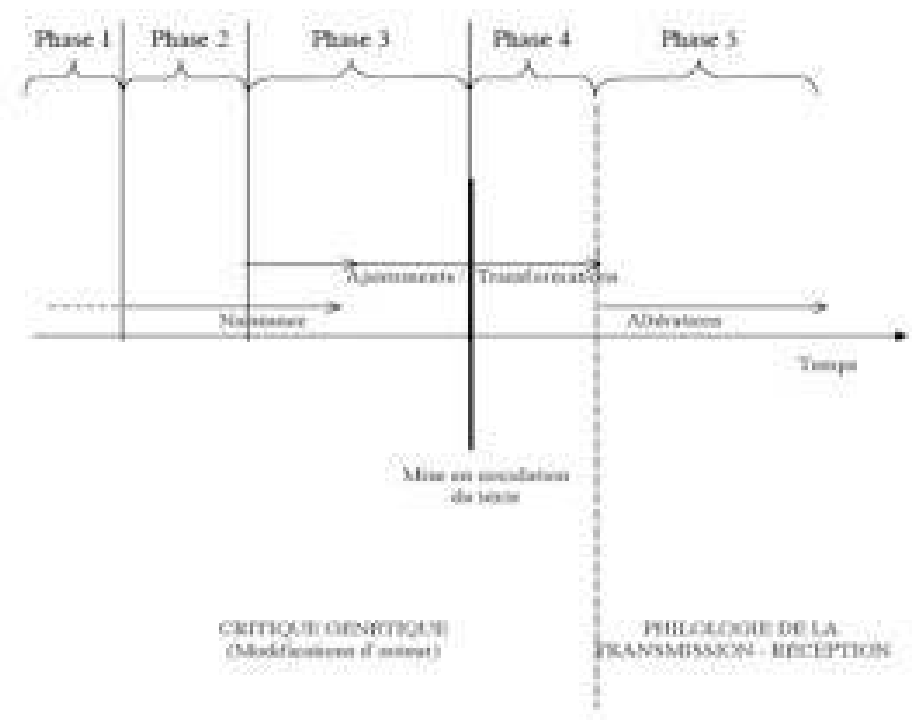

Cette parenté profonde entre toutes les formes de variation textuelle trouve sa parfaite illustration dans le logiciel MEDITE, développé par J.-

6. J.-G. Ganascia \& J.-L. Lebrave, « Trente ans de traitements informatiques des manuscrits de genèse » dans Critique génétique. Concepts, méthodes, outils, $\mathrm{O}$. Anokhina \& S. Pétillon (éd), Imec éditeur, 2009, p. 68-82. 
G. Ganascia et J. Bourdaillet. Il compare entre eux des états textuels ramenés à la forme la plus désincarnée que ceux-ci puissent prendre, puisqu'il s'agit de suites de caractères " en mode texte ", c'est-à-dire dans lesquelles les graphèmes et les ponctuations sont réduits à une pure idéalité. Rien d'étonnant donc à ce qu'il ait constitué un outil particulièrement bien adapté au cas des textes en variation de Ramuz dont J.-M. Adam parle dans son article?

Ce n'est pas à ce niveau textuel qu'il faut appréhender la singularité des manuscrits de travail. Ce qui oppose de manière radicale les dossiers génétiques aux textes dans l'idéalité que leur confère l'imprimé ou la « belle copie », c'est l'omniprésence de la substance graphique : il n'y a pas de différence de nature entre les variations textuelles que J.-M. Adam identifie dans les états successifs des Contes de Perrault et les substitutions que j'ai construites à partir des brouillons de la Lutezia de Heine. En revanche, la manière dont ces dernières se donnent à voir dans la substance graphique des brouillons est sans équivalent direct dans les cas analysés par J.-M. Adam. À cette différence s'ajoute aussi, bien sûr, une différence de statut énonciatif, qui tient à l'originalité de l'écriture manuscrite moderne comme mode de " composition » au sein de l'histoire longue des modes de multiplication et de circulation des textes.

En résumé, en tant qu'elle est une poétique des transitions entre états, la critique génétique est bien la fille de la philologie, ou plutôt une proche parente de la " nouvelle philologie ${ }^{8}{ }^{8}$, mais aussi de la variantistica de Contini ${ }^{9}$. Mais ceci ne rend pas encore pleinement justice à la singularité des matériaux sur lesquels elle travaille et ne la rapproche pas encore beaucoup d'une science des processus eux-mêmes.

\section{De la variance médiévale à l'avant-texte et au-delà}

L'importance primordiale prise par la substance graphique individuée est à mettre en relation avec l'évolution des pratiques de composition dont on peut situer le point d'aboutissement provisoire à la fin du XXe siècle. En quelques siècles s'est mise en place une forme d'écriture privée dans laquelle la performance a perdu le rôle fondamental qu'elle jouait jusque

7. Cf. I. Fenoglio, J.-G. Ganascia, J.-L. Lebrave, « Manuscrits, genèse et documents numérisés. EDITE : une étude informatisée du travail de l'écrivain », Document numérique, vol. 8, $\mathrm{n}^{\circ}$ 4/2004 ; J. Bourdaillet, J.-G. Ganascia, J.-L. Lebrave, "Topologie et génétique textuelles : un dialogue médié par la machine », Lexicometrica, "Topographie et topologie textuelles », numéro spécial, sous presse ; R. Mahrer, « La Génétique Assistée par Ordinateur : Medite au banc d'essai ou Du tout neuf pour le Tout-Vieux », Genesis n²7, 2006.

8. Comme le soulignait déjà B. Cerquiglini en 1989. Cf. B. Cerquiglini, Éloge de la variante. Histoire critique de la philologie. Paris, Éd. du Seuil, 1989.

9. G. Contini, Varianti e altra linguistica. Una raccolta di saggi (1938-68), Turin, Einaudi, 1970. 
là. Pour le dire d'un mot, le crissement de la plume sur le papier a remplacé le grain de la voix, et ce déplacement a modifié de manière fondamentale le statut de la variation. Jusqu'à cet avènement de l'écriture moderne, le texte devait être prononcé pour accéder à la complétude d'un échange abouti entre celui qui produit le discours et ceux qui l'écoutent. Cette performance nécessaire entraînait presque mécaniquement la prolifération de la variation, même si les protagonistes étaient persuadés du contraire, comme on s'en est rendu compte lorsqu' on a pu comparer des enregistrements d'épopées orales réalisés à quelques années $\mathrm{d}^{\prime}$ intervalle $\mathrm{e}^{10}$ : on a constaté dans ce cas que la fixité absolue attribuée de manière unanime au texte du récit par les récitants et le public n'avait pas le caractère littéral que lui confère l'écrit : en réalité, l'épopée n'était jamais chantée deux fois de manière identique. On sait depuis Zumthor que la mouvance médiévale est intimement liée à ce bruissement continu de la langue qui exprimait la nécessité pour le texte d'être performé, et donc interprété. A notre époque, cette performance jadis omniprésente n'a survécu qu'au théâtre, et, dans une bien moindre mesure, dans les lectures publiques que les écrivains font parfois de leurs œuvres.

J'ai esquissé ailleurs le parcours qui, à travers la lente montée en puissance de la lecture silencieuse, a contribué à rendre l'écrit indépendant de sa mise en œuvre dans une performance ${ }^{11}$. L'augmentation exponentielle du nombre de manuscrits de travail conservés à partir du XVIII ${ }^{\mathrm{e}}$ siècle a certainement des causes multiples et hétérogènes. Avec toute la prudence qui s'impose sur une question difficile et mal connue, on peut affirmer que l'une de ces causes est une modification des pratiques de composition. Pour rester dans la problématique que j'ai développée ici, cette évolution affecte avec la même force les deux versants de l'acte de communication, en transformant l'écriture et la lecture, la production et la réception, en activités privées. Le scripteur produit son texte dans l'isolement de son cabinet de travail ${ }^{12}$, et nul lecteur n'est censé être témoin du processus de production du texte. Symétriquement, la performance silencieuse et passive qui échoit au lecteur se fait en l'absence du scripteur, qui ne connaît les réactions de son public que sous une forme indirecte et médiée.

On sait désormais que le « préjugé philologique » du texte invariant, clos, fini et achevé a partie liée avec cette évolution. Je voudrais en

10. Albert B. Lord, The Singer of Tales, Harvard University Press, Cambridge, Mass., 1964.

11. Cf. Jean-Louis Lebrave, «La production littéraire entre l'écrit et la voix». M. Contat et D. Ferrer (eds), Pourquoi la critique génétique? Méthodes et théories. CNRS Éditions, 1998, pp. 169-188.

12. Même si certains troquent celui-ci pour une table de café : dans ce cas, c'est l'acte d'écriture qui est mis en scène, non le processus de production lui-même, qui reste caché aux spectateurs éventuels. 
montrer ici une conséquence en la rattachant à la prolifération de la substance graphique dont j'ai souligné l'importance. Aussi longtemps que production et performance sont inséparables, la variation est partout. C'est le cas pour la littérature du Moyen-Âge. Ce l'est encore pour les Contes de Perrault, comme le montre bien J.-M. Adam dans ce volume. À l'opposé de cette mouvance, la philologie du XIXe siècle nous a habitués à postuler l'invariance supposée du texte, dont le corollaire serait l'existence d'un sens lui aussi invariant. Les manuscrits de travail se trouvent à bien des égards entre les deux, et leur variation foisonnante s'oppose en tout point à la fixité attribuée au texte. L'évolution a eu pour effet de faire passer d'une variation liée à la pluralité des énonciateurs d'une même œuvre à l'unicité d'un seul auteur qui prend en charge la variation au cours du processus de production. C'est dans le brouillon que le texte est variant, et non plus à travers ses différentes performances.

On peut donc dire que les brouillons gardent, à la manière du sillon laissé par l'instrument d'écriture sur la feuille de papier, la trace en creux de la variance qui caractérise la genèse. Je souscris volontiers à la thèse très saussurienne défendue par J. Peytard et J.-M. Adam, qui posent que le sens naît de la variation. Il en résulte une conséquence intéressante lorsqu'on l'applique aux manuscrits de travail. S'ils sont le lieu de la variation, telle qu'elle s'exprime à travers la prolifération de la substance graphique sur laquelle j'ai insisté tout à l'heure, alors ils sont aussi un lieu privilégié du sens, qui se niche dans les creux de l'inscription des ratures dans le brouillon. En montrant ainsi comment le sens advient au texte dans les brouillons, la linguistique apporte une réponse originale à la question, souvent débattue, de savoir si la critique génétique est ou non une critique.

Reste que l'évolution des techniques de composition n'est pas achevée. Avec le traitement de texte, la mutation actuelle fait disparaître la substance graphique des manuscrits, et fait disparaître en même temps la trace des opérations d'écriture en donnant à voir des textes dont la surface est indéfiniment lisse et achevée. L'extinction de la variation n'est qu'apparente : elle est même beaucoup plus proliférante encore que dans le cas des manuscrits de travail. La génétique des transitions [Je trouve l'expression intéressante, peut-être mériterait-elle un petit développement] entre états successifs a donc de beaux jours devant elle. Il n'est pas certain qu'on puisse dire la même chose de l'élucidation du processus génétique lui-même, puisque les traces qu'il laisse derrière lui disparaissent ou changent de nature. Mais peut-être n'est-ce plus la tâche de la linguistique... 